\title{
Evaluation of Video Transmission of MAC Protocols in Wireless Sensor Network
}

\author{
Maulidin $^{1}$, M. Mahmuddin ${ }^{1}$, L.M Kamarudin ${ }^{2}$ \\ ${ }^{1}$ InterNetwork Research Laboratory, School of Computing, UUM College of Arts and Sciences, \\ Universiti Utara Malaysia, 06010 Sintok, Kedah, Malaysia \\ ${ }^{2}$ School of Computer and Communication Engineering, Universiti Malaysia Perlis, Malaysia. \\ maulidin84@gmail.com
}

\begin{abstract}
Wireless Sensor Network (WSN) is a wireless network which consists of sensor nodes scattered in a particular area which are used to monitor physical or environment condition. Each node in WSN is also scattered in sensor field, so an appropriate scheme of MAC protocol should have to develop communication link for data transferring. Video transmission is one of the important applications for the future that can be transmitted with low aspect in side of cost and also power consumption. In this paper, comparison of five different MAC WSN protocol for video transmission namely IEEE 802.11 standard, IEEE 802.15.4 standard, CSMA/CA, Berkeley-MAC, and Lightweight-MAC protocol are studied. Simulation experiment has been conducted in OMNeT++ with INET network simulator software to evaluate the performance. Obtained results indicate that IEEE 802.11 works better than other protocol in term of packet delivery, throughput, and latency.
\end{abstract}

\section{Index Terms-WSN; MAC Layer Protocol.}

\section{INTRODUCTION}

Wireless Sensor Networks (WSN) can be considered as worthy capable solution for the future of green technology. In the last decade, WSN has been a world-wide interest due to their wide range of potential applications as following: environment monitoring [12] target tracking [7] surveillance [6]. The introduction of Complementary Metal Oxide Semiconductor (CMOS) based camera nodes technology allowed the capability to transmit video and image in WSN application. These technologies improve WSN real-time application such as surveillance and monitoring, due to their potential to collect visual information which is not available with traditional WSN nodes.

In WSN real-time application, it requires comparatively high bandwidth utilization, throughput, and bounded end-to-end delay. Therefore, the effective design in WSN medium access control (MAC) protocols has anticipated be more challenging task and results for supporting real-time communication in WSN. This is because the MAC protocol provides a mechanism to control channel access that allows nodes to communicate.

Lately, numerous studies performance MAC on different WSN have different design requirement [2]. There are which are to be considered before designing MAC protocol like traffic generation density, packet size and mobility that affect the performance. A careful design consideration should be given of these factors before a practical wireless sensor network is realized.

This paper presents appraises the performance evaluation of five MAC layer protocols through simulation and analysis. Discrete event simulator OMNeT++with INET framework has been chosen for experiments MAC protocol behaviour. The rest of this study is structured as follows. Section-II delivers an overview of the MAC protocol. In Section-III, simulation results are presented. Eventually, in Section -IV concludes this paper.

\section{MAC PROTOCOLS}

The MAC layer is primarily responsible for regulating access to the shared medium and ensuring there is no nodes interfering with other communications. As per Open Systems Interconnection model (presented in Fig.1), MAC layer may refer to the sub-layer of data link layer (DLL). MAC layer protocols for WSNs expected be energy efficient to maximize network lifetime, scalable to network size, and should adjust to changes network as a major priorities [5]. This section covers some popular MAC layer protocols for WSN.

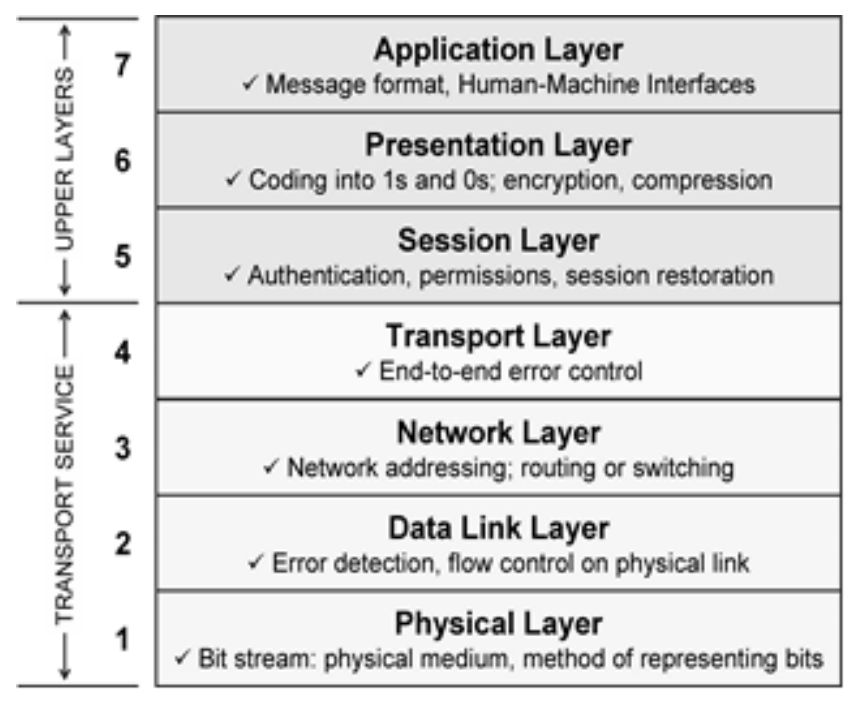

Figure 1: The OSI Network model

\section{A. IEEE 802.11}

IEEE 802.11 is standard basis MAC for WLAN (Wireless local area network). The MAC sub layer of the IEEE 802.11, 
presented Distributed Coordination Function (DCF) technique use CSMA/CA, Point Coordination Function (PCF) technique use Access Point, and also Hybrid Coordination Function (HCF) technique enhance DCF and PCF. IEEE 802.11 defines two sensing processes that are used to improvement access the medium such as; namely, Physical carrier sensing (PCS) and Virtual Channel Sensing (VCS) [4]. It uses carrier detecting and randomized back-offs to prevent collisions of data packet. The main appearances of the 802.11 WLAN technologies are flexibility, simplicity, and the effectiveness of cost rate [1].

\section{B. IEEE 802.15 .4}

IEEE 802.15.4 is designed for low rate wireless personal area networks. This attribute enables small, energy-efficient, low cost solutions to be instigated for a varied range of mechanism of devices. It describes the medium access control (MAC) and the physical layer (PHY) sub layer specifications for low-data rate wireless connectivity with firm, portable, and moving devices with very limited battery. The 802.15.4MAC operating in beacon enable and non-beacon enable.

\section{C. $C S M A / C A$}

IEEE 802.15.4 is designed for low rate wireless personal area networks. This attribute enables small, energy-efficient, low cost solutions to be instigated for a varied range of mechanism of devices. It describes the medium access control (MAC) and the physical layer (PHY) sub layer specifications for low-data rate wireless connectivity with firm, portable, and moving devices with very limited battery. The 802.15.4MAC operating in beacon enable and non-beacon enable.

\section{D. $B-M A C$}

This protocol employs static wakeup preamble sampling structure to decrease the duty-cycle and reduce idle listening [13]. In order to gain low power consumption, B-MAC combines both techniques of CSMA and Low Power Listening (LPL). This protocol provides a good low power operation, effective collision avoidance, efficient channel utilization.

\section{E. $L-M A C$}

Lightweight MAC (L-MAC) is a TDMA based MAC protocol uses a disseminated algorithm for slot selection mechanism based on two-hop neighbor information [8]. Each slot consists two element parts: control message and data message period. Upon receiving message receiver will decide to stay awake or not. By combining message from all neighboring node is able to determine unoccupied slots. Process starts from base station, during each frame it continuous throughout network.

Due to the wireless communication and adequate resources and tough challenges in WSN, the MAC protocol efficiency is one of the most essential factors that require to be considered before designing several aspects of applications. In wireless sensor network, for designing high quality MAC protocol, the following characteristics such as energy consumption, packet delivery, throughput, and latency are required to be considered [5].

This work, mainly concentrate only on three attributes performance as states in. a) Packet Delivery Ratio: is the ratio of received packet over sent packet per unit time in the network.

b) Throughput: the total rate of packets received per unit frame by the sink node.

c) Latency: the amount of time delay between the sending of packet to the time when the packet reaches the sink node.

\section{OBSERVATION AND RESULTS}

\section{A. Simulation parameters}

This work presents various MAC protocols for WSN using IEEE 802.11, IEEE 802.154, CSMA/CA, Berkeley-MAC and Lightweight-MAC. A simulation experiment was performed by using OMNeT++-4.6 versions along with INET-3.0 framework to study the performance of the protocol. Table 1 summarizes the system parameters used for the simulations. After running the simulations, the performance matrices: packet delivery ratio, throughput, and latency are observed.

Table 1

Parameters use for the simulations

\begin{tabular}{ll}
\hline No of hosts & 10 \\
No of Sink & 1 \\
Application Type & Video Stream \\
Mobility Type & Stationary (8 host) and Rectangle (2 host) \\
Mobility Area & $(0$ meter,0 meter $)$ \\
Mobility Target & $(500$ meter,300 meter $)$ \\
Constraint Area & $(500$ meter,300 meter $)$ \\
Video Size & $100 \mathrm{MiB}$ \\
Packet Length & $1024 \mathrm{~B}$ \\
Radio Type & APSKScalarRadio \\
Traffic Generator & $200 \mathrm{~KB} / \mathrm{s}, 400 \mathrm{~KB} / \mathrm{s}, 600 \mathrm{~KB} / \mathrm{s}, 800 \mathrm{~KB} / \mathrm{s}, 1000 \mathrm{~KB} / \mathrm{s}$ \\
Simulation Time & $200 \mathrm{~s}$ \\
\hline
\end{tabular}

\section{B. Result}

In this section, we provide head to head comparison packet delivery ratio, throughput, and latency of different MAC protocols for video transmission in WSN. The result show the average of 5 simulations run with the same setting, but with different seed for the random number generators.

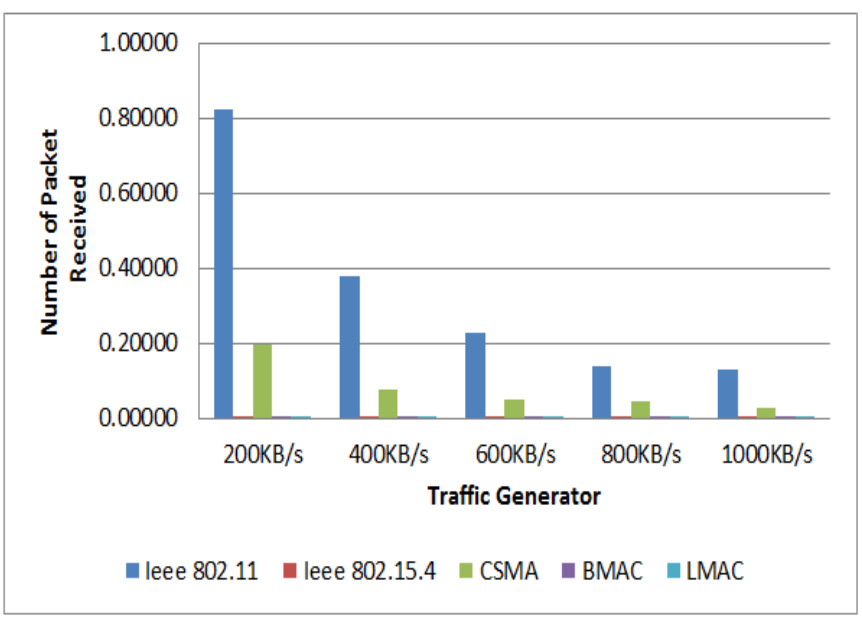

Figure 2: Means of Packet Delivery 


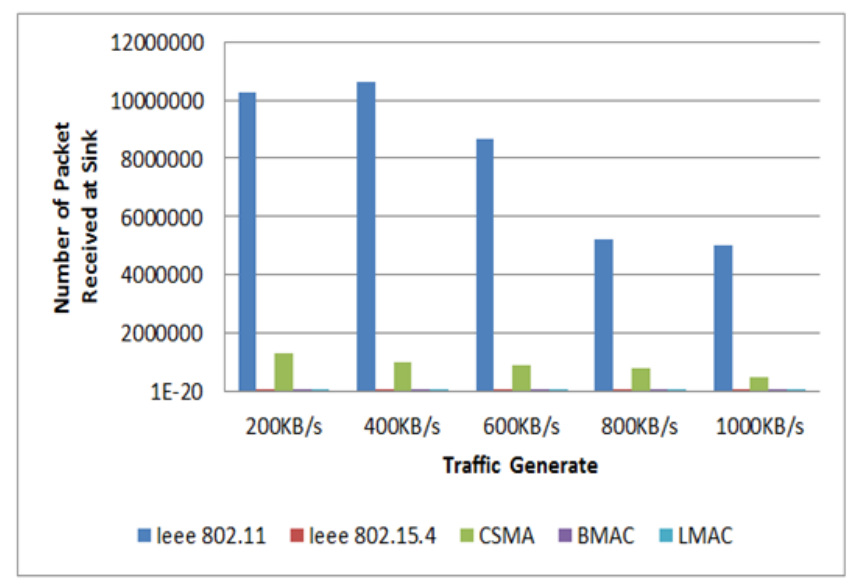

Figure 3: Means of Throughput

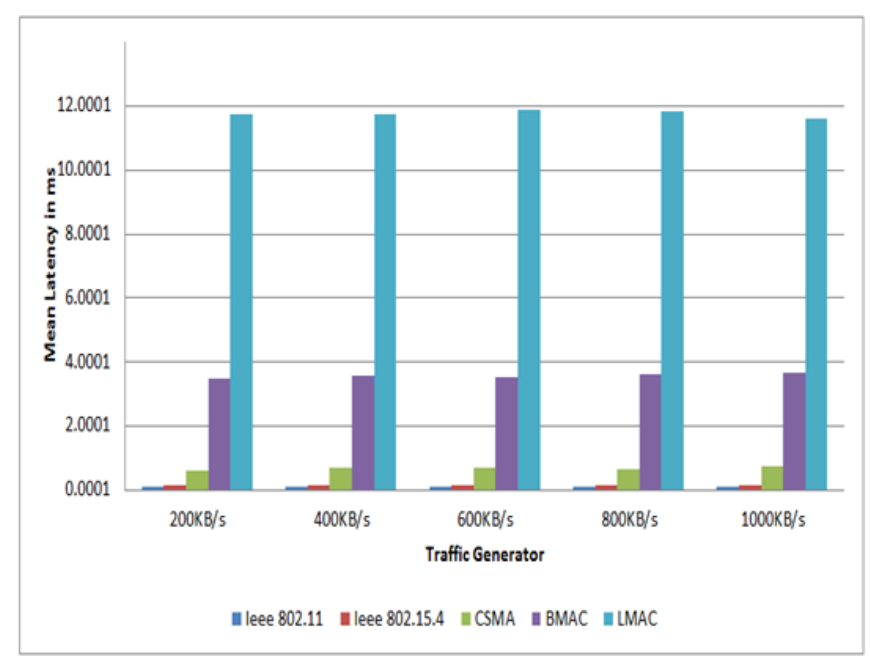

Figure 4: Means of Latency

To evaluate the packet delivery ratio, the total amount of packets that are received at the destination is divided with the total number of packets that are sent by the sender. As shown in Figure 2, the comparison packet delivery ratio vary for the entire MAC when traffic rate increase. The curve clearly show that with traffic generation rate was increasing, packet delivery ratio may decrease. Under this scenario IEEE 802.11 performs well with $82.56 \%$ average of PDR and CSMA/CA MAC performs $19.69 \%$. While IEEE 802.15.4, Berkeley-MAC, and Lightweight-MAC can perform well with video transmission. Thus, this kind of network not feasible for sensor networks which has multimedia system that rely transmission of video via wireless medium.

Figure 3 represent the throughput comparison between IEEE 802.11, IEEE 802.15.4, CSMA/CA, Berkeley-MAC, and Lightweight-MAC network. MAC throughput signified by total quantity of data delivered to the sink. As the number traffic rate increase, the probability of successful transmission decreases. In this scenario again IEEE 802.11 exhibits the best overall performance for video transmission compares to others protocol. This MAC protocol can deliver well for video transmission until $400 \mathrm{~kb} / \mathrm{s}$.

Finally, Figure. 4 show the behaviour average packet latency in five MAC protocols for various traffic loads. Average packet latency depends on route discovery latency, besides delays at each hop and the number of hops. The trend of the shown curves clearly illustrates that IEEE 802.11 show good performance in term of average latency compare to other MAC protocol. Lightweight-MAC protocol show bad performance compare to other protocols.

Based on our simulation of video transmission over MAC WSN, show that when designing MAC WSN should be considering various factor consist of number of hosts, mobility type, host position, and traffic generations rate. Besides that, cross layer design and multi-channel approach may be needed for optimized design of supporting video transmission in wireless sensor network.

\section{CONCLUSION}

MAC layer for wireless sensor network is a challenging because of limited power in battery and bandwidth. In order to meet these requirements, a good MAC protocol for WSNs should be select carefully. It can be concluded that in a scenario for video transmission, IEEE 802.11 is the very much applicable and followed by CSMA/CA, IEEE 802.15.4, Berkeley-MAC, and Lightweight-MAC. According to the evaluation of this MAC performance, we observe that majority of the protocols depend on number of sensor nodes, mobility pattern, playground size and traffic load. Design trade-offs and open research issues that can be pursued for further investigation in the field of Video Transmission in WSN at MAC layer.

\section{REFERENCES}

[1] A. Abbas. M.H. Ibrahim, and M.H. Osama. "Fairness and Quality of Service Issues and Analysis of IEEE 802.11e Wireless LAN," IN International Journal of Electrical, Computer, Energetic, Electronic and Communication Engineering, vol.4, no.1, pp.163-169. 2010.

[2] A.A. Ahmad. Alkhatib and S.B. Gurvinder. "MAC Layer Overview for Wireless Sensor Networks", Proc. of IPCSIT International Computer Networks and Communication Systems Conference Proceedings, CNCS 2012, Vol.35, Singapore. 2012.

[3] M. Bertocco. G. Gamba. S. Alessandro. \& S. Vitturi. "Performance measurements of CSMA/CA-based wireless sensor networks for industrial applications," In Instrumentation and Measurement Technology Conference Proceedings, IMTC 2007, IEEE, pp. 1-6. IEEE, 2007.

[4] C.E. Campbell. K.K. Loo. H.A. Kurdi., \& S. Khan. "Comparison of IEEE 802.11 and IEEE 802.15. 4 for future green multichannel multi-radio wireless sensor networks," International Journal of Communication Networks and Information Security (IJCNIS), vol.3, no.1.2011.

[5] I. Demirkol., C. Ersoy., \& F. Alagöz. "MAC protocols for wireless sensor networks: a survey", Communications Magazine, IEEE, vol.44, no.4, pp.115-121. 2006.

[6] Q. Guifang. S. Guangming. Z. Ying. Z. Jun. P. Jin. "Lifetime Optimization of an Indoor Surveillance Sensor Network Using Adaptive Energy-Efficient Transmission." International Journal of Distributed Sensor Networks, pp.1-12. 2015.

[7] W. Guojun. M. Z. Alam Bhuiyan. C. Jiannon. Jie Wu. "Detecting Movements of a Target Using Face Tracking in Wireless Sensor Networks," in Parallel and Distributed Systems, IEEE Transactions on, vol.25, no.4, pp.939-949. 2014.

[8] L. V. Hoesel and P.J.M. Havinga. "A lightweight medium access protocol (LMAC) for wireless sensor networks: Reducing preamble transmissions and transceiver state switches," pp, 205-208. 2004.

[9] IEEE, (2001). "IEEE Standards for local and metropolitan area networks: overview and architecture," IEEE Std 802-2001 (Revision of IEEE Std 802-1990).

[10] IEEE Standard for Wireless LAN Medium Access Control and Physical Layer Specification, STD 802.11-2007. 
[11] IEEE, (2006).std. 802.15.4, Part. 15.4: Wireless Medium Access Control (MAC) and Physical Layer (PHY) Specifications for Low-Rate Wireless Personal Area Networks (LR-WPANs), IEEE standard for Information Technology, IEEE-SA Standards Board, Sept.

[12] K. Loizou. E. Koutroulis. D. Zalikas. G. Liontas. "A low-cost sensor based on Time-Domain Reflectometry for water level monitoring in environmental applications," in Environment and Electrical Engineering
(EEEIC), 2015 IEEE 15th International Conference on, pp.261-266. 2015.

[13] J. Polastre. J. Hill. \& D. Culler. "Versatile low power media access for wireless sensor networks," In Proceedings of the 2nd international conference on Embedded networked sensor systems, ACM, pp.95-107. 2004. 